\title{
Growth and habit modification of sodium chlorate crystals
}

\author{
V SURENDER and K KISHAN RAO \\ Department of Physics, Kakatiya University, Warangal 506009, India \\ MS received 12 August 1992; revised 5 January 1993
}

\begin{abstract}
Single crystals of sodium chlorate were grown in the presence of selective impurities. The morphology of these crystals changed completely from cubic to tetrahedral form. Experiments were performed in the presence of different concentrations of impurities to ascertain their role in modifying the habit of these crystals. The presence of impurity in the solution was found to affect the habit of the crystals, although it does not appear to enter into the lattice.
\end{abstract}

Keywords. Crystal growth; sodium chlorate.

\section{Introduction}

Sodium chlorate belongs to the cubic tetrahedral class. The morphology of these crystals when grown from pure aqueous solutions is cubic-bounded by $\{100\}$ faces whereas isomorphous sodium bromate crystals grow in tetrahedral form bounded by $\{111\}$ faces. Considerable work has been reported on the effect of impurities on growth morphology of various crystals (Kolb and Comer 1945; Butchart and Whetstone 1949). Buckley (1951) pointed out that the growth morphology of $\mathrm{NaClO}_{3}$ could be modified to tetrahedral form when grown in the presence of some selective impurities. Of the impurities, only sodium thiosulphate and borax modify the morphology at a lower concentration. Borax was chosen as the selective impurity for the present work.

\section{Experimental}

In the present growth experiments, saturated solution of $\mathrm{NaClO}_{3}$ was prepared in bulk at $35^{\circ} \mathrm{C}$ and distributed into various jars. Already prepared borax solution was added appropriately to the pure solution in the molar ratio varying from $0: 50$ to $1: 50$.

After ascertaining the saturation state of the solutions, small $\{100\}$ crystals obtained from pure solution were introduced into the solutions containing different concentrations of borax for growth. All growth experiments were carried out by slow evaporation process at $35^{\circ} \mathrm{C}$. Transparent crystals with well-defined faces were obtained in 10 days.

\section{Results and discussion}

Figure 1 shows the crystals obtained from different jars containing various concentrations of borax. It can be clearly seen here that the habit of the crystals changes from $\{100\}$ to tetrahedral form bounded by $\{111\}$ faces. As the borax concentration increases, new triangular faces start appearing at the corners of the cube as shown in figure 2. These triangular faces grow in size as the borax 


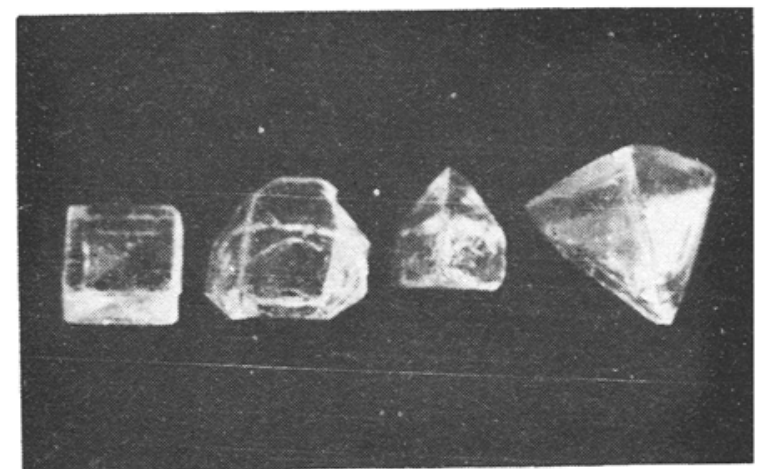

Figure 1. Observed habit modification in $\mathrm{NaClO}_{3}$ in the presence of borax in molar ratios, $0: 50,1: 150,1: 75$ and 1:50.

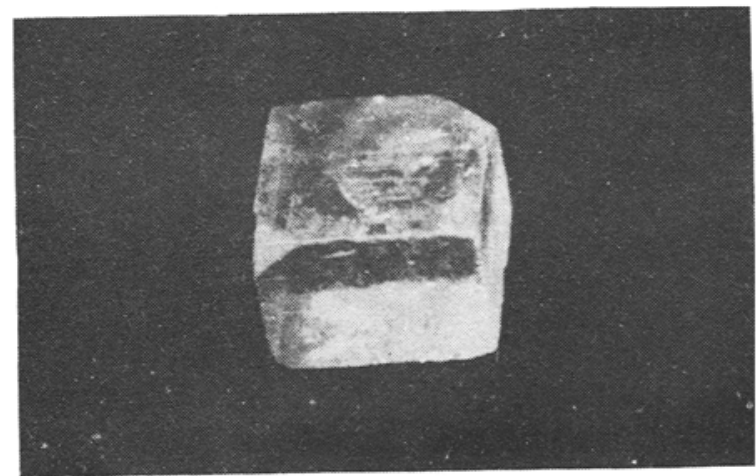

Figure 2. Appearance of triangular faces on cubic $\mathrm{NaClO}_{3}$ crystal.

concentration increases. Finally $\{100\}$ faces are eliminated and crystals are bounded by $\{111\}$ faces only. It has been observed that total transition to $\{111\}$ takes place when borax is present in the molar ratio of 1:50 in the solution.

Further, from the same set of jars, the crystals collected from the jars in different batches were studied. The crystals collected in the first phase are called crop I. Once again new $\{100\}$ seeds were introduced into the jars and the solutions were further left for evaporation and subsequently crops II and III were collected. It is interesting to note that some crystals with $\{111\}$ faces were obtained even in the solution which initially had lower concentrations of borax.

It can be seen from the above two sets of experiments that the habit of $\mathrm{NaClO}_{3}$ crystals is modified when borax is present in the solution in the molar ratio 1:50 and even in the solution having a lower concentration of borax, the habit is modified in crops II and III crystals. In order to find out whether borax is entering the lattice or not, the crystals were subjected to chemical analysis, powder XRD, spectral and hardness studies. None of them revealed the presence of borax in the lattice. The habit modification in the case of crops II and III crystals in lower borax concentration could be because as the crystals are withdrawn from the solution, the concentration of borax increases.

It is interesting to note from figures 2 and 3 that only alternate corners of the cube 


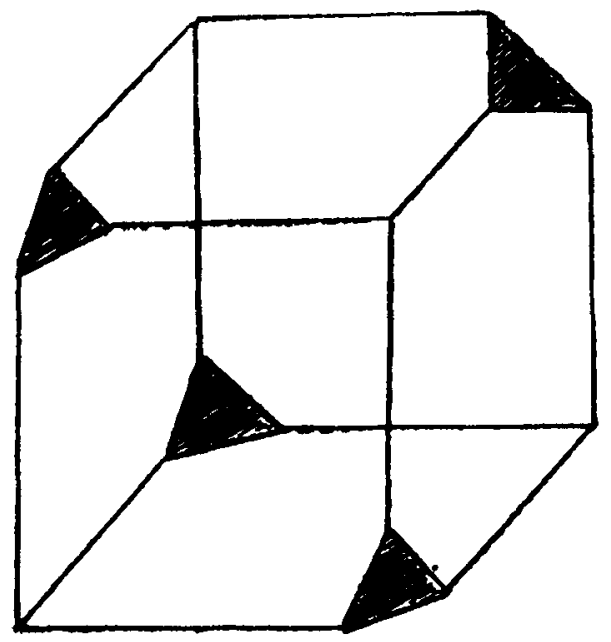

Figure 3. Line diagram showing the appearance of (111) faces.

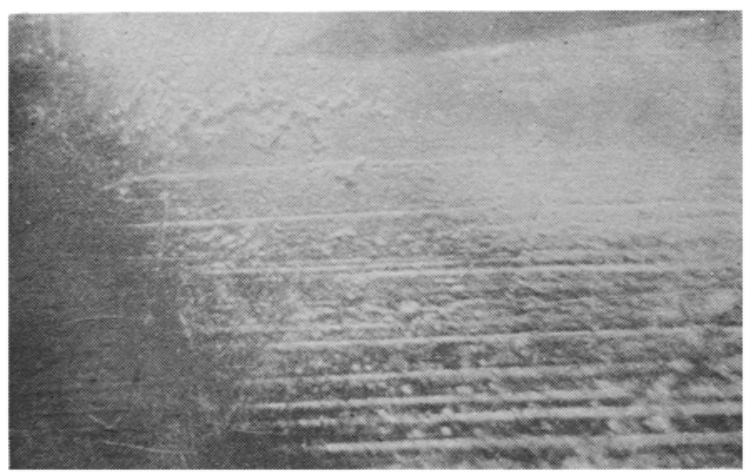

Figure 4. Step pattern along $\langle 110\rangle$ direction on (111) faces of $\mathrm{NaClO}_{3}$.

develop into triangular faces. Recently, Tsukamoto and Sunagawa (1990) have observed on $\mathrm{KCl}$ that all the eight corners of the cube develop into $\{111\}$ faces in the presence of $\mathrm{Pb}$ impurities. As $\mathrm{NaClO}_{3}$ crystals belong to non-centrosymmetric system, the atomic arrangement on $\{111\}$ and its opposite $\{\overline{1} \overline{1} 1\}$ faces being different, it appears that only one set of $\{111\}$ faces is observed to grow.

Immediately after the growth, the crystals were dried and transferred to reflection microscope to observe growth patterns. No growth spirals were observed on $\{100\}$ and $\{111\}$ surfaces. But steps parallel to $\langle 110\rangle$ direction were observed only on $\{111\}$ faces as shown in figure $4 .\{100\}$ and $\{111\}$ crystals were chemically etched with suitable etchants (Kishan Rao and Sirdeshmukh 1978) to study dislocation distribution. Figure $5(a, b)$ shows the etch pit pattern on $\{100\}$ and $\{111\}$ faces of these crystals. The distribution is random on $\{100\}$ faces whereas the concentration of triangular pits is greater along the $\langle 110\rangle$ edges on $\{111\}$ faces.

The present studies show that the habit change of $\mathrm{NaClO}_{3}$ from cubic to tetrahedral form in the presence of borax could be due to hindrance of $\langle 110\rangle$ steps on $\{100\}$ 

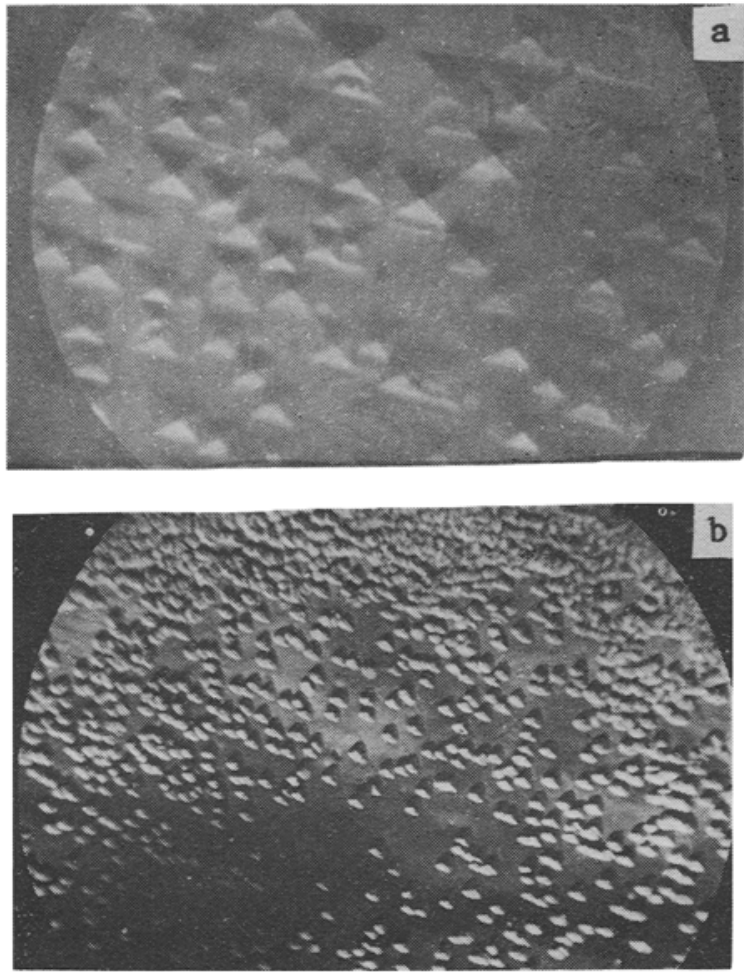

Figure 5. Etch pit pattern on (a) (100) surface and (b) (111) surface.

faces by borax. Perhaps this reduces the advancing rate of growth layer in this direction. It resuits in the piling up of one set of $\langle 110\rangle$ steps on $\{100\}$ faces. Hence, $\{111\}$ faces appear at the preferential corners, they grow into a tetrahedral form in a few days.

\section{Acknowledgements}

The authors thank Prof. D B Sirdeshmukh for useful discussions and suggestions. The authors are grateful to the referee for numerous suggestions.

\section{References}

Buckley H E 1951 Crystal growth (Wiley: New York) p. 554

Butchart A and Whetstone J 1949 J. Discuss. Faraday Soc. 5255

Kolb H J and Comer J J 1945 J. Am. Chem. Soc. 67894

Kishan Rao K and Sirdeshmukh D B 1978 J. Cryst. Growth 44533

Tsukamoto L K and Sunagawa I 1990 J. Cryst. Growth 99150 